\title{
Introduction to the Special Issue: Concussion Management in Sport
}

\author{
Maureen R. Weiss
}

I am pleased to present this special issue of Kinesiology Review on concussion management in sport, a ubiquitous source of concern in the media, among health care professionals, and in the scholarly literature. Even as I write this introduction, the national news is covering the story of a former high school football player who filed a class-action lawsuit against his state's high school association for "systemic failure to protect players" from the danger of concussions and long-term negative effects on the developing brain. Over the last decade, there have been significant advances in scientific knowledge regarding concussion and the development of policy statements regarding assessment, diagnosis, recovery, and return to play after a concussion. In this special issue, authors of five articles extensively review sport-related concussion from the viewpoints of pediatrics, sports medicine, psychology, philosophy, and athletic training. This interdisciplinary approach is important because concussion involves multifactorial and complex processes related to risk, assessment, care, and outcomes. For me, two common themes in particular emerge: (a) a need for a holistic approach to assessing and treating concussion (i.e., treat the whole person, not the injury), and (b) the importance of collaboration and communication among health care providers, families, schools, and kinesiology scholars to effectively treat and aid recovery from sportrelated concussion.

In the first article, Tamara C. Valovich McLeod, Megan N. Houston, and Cailee E. Welch discuss critical factors specific to children's and adolescents' susceptibility to, and recovery from, sport-related concussion. The authors provide data on prevalence and escalation of concussions in pediatric populations and address developmental issues regarding management and recovery. Given the lack of knowledge about concussion and low frequency of reporting symptoms by those who oversee youth sport, the authors emphasize the need to educate coaches, parents, league officials, and the youth participants themselves. Valovich McLeod and colleagues

Weiss is the editor of Kinesiology Review; Weiss is also a professor with the School of Kinesiology and an adjunct professor with the Institute of Child Development, both at the University of Minnesota, Minneapolis, MN. review legislation and policies by state interscholastic associations, followed by a discussion of preparticipation examinations and age-appropriate assessments of concussion. They contend that concussion management should consider the impact on health-related quality of life and the need for cognitive and physical rest and academic accommodations at school. The authors emphasize collaborative communication among health care professionals, school personnel, coaches, and family members to ensure safe and appropriate actions for return to school and physical activity following concussion.

In the second article, Kevin Guskiewicz and Elizabeth Teel present a multifaceted approach to the clinical diagnosis and management of sport-related concussions. Their focus is on using objective tests by trained professionals to accurately diagnose and treat concussions. They provide detailed information on assessment tools specific to sideline evaluation (e.g., symptom checklists, postural sway), clinical and laboratory evaluation (e.g., neuropsychological testing, sensory tests, gait measures), and neurophysiological evaluation (e.g., electroencephalography, event-related potentials, magnetic resonance imaging). Guskiewicz and Teel highlight the importance of collaboration among sports medicine personnel and kinesiology researchers in many subdisciplines, including biomechanics, exercise physiology, motor control, and sport and exercise psychology. This interdisciplinary approach will expand knowledge of risk, assessment, management, and recovery of sport-related concussion that ultimately translates into interventions that optimize athletes' health and well-being.

In the third paper, Diane M. Wiese-Bjornstal, Andrew C. White, Hayley C. Russell, and Aynsley M. Smith provide an in-depth review of the psychological, psychiatric, and psychosocial factors that affect risks, treatment, and consequences of sport-related concussion. They clarify psychological factors as thoughts, feelings, and behaviors related to sport concussions, such as cognitive appraisals, emotions, and coping strategies; psychiatric factors include prevention and treatment of mental illness associated with concussion, such as depression and anxiety disorders; and psychosocial factors refer to physical and social environmental attributes that affect individuals at risk for, or recovering from, concussion (e.g., team norms, interpersonal communication with 
coaches and athletic trainers). In reviewing the empirical research, Wiese-Bjornstal and colleagues situate studies of psychological aspects of sport concussion within a dynamic and integrated model of sport injury, which highlights preinjury psychological risk factors, postinjury psychological response processes, and postinjury psychological care. The model provides an evidence-based framework for understanding predisposing risk factors (e.g., personality); postinjury rehabilitation (e.g., personal and situational factors); and psychological care using a holistic approach to assessment (behavioral, cognitive, affective, physiological), providers (neuropsychologists, psychiatrists, clinical psychologists, school psychologists, sport psychologists), and interventions (education, social support, cognitive behavior therapy).

The fourth article, authored by Michael J. McNamee, Bradley Partridge, and Lynley Anderson, focuses on philosophical and ethical considerations in sport-related concussion management. They critically review some conceptual inconsistencies in definitions and prevalence of concussion in the scientific literature, as well as in recommendations by medical consensus statements on the diagnosis and management of concussion in sport. This review leads to a discussion of ethical issues related to concussion in child and adolescent populations, the potential need and justification for paternalistic attitudes, the danger of conflicting and competing interests, and confidentiality of health information.

The final paper is by Charles R. Thompson, head athletic trainer at Princeton University, and is a translational piece focused on the role of the athletic trainer as a critical medical team member in the care of college student-athletes. He describes the historically-prevalent sports medicine management model at colleges, which situates the athletic trainer as an employee of the athletic department, and he contrasts this type of model to one in which the athletic trainer is hired by the institution's health service unit. This latter "medical model", present in only a few universities, eliminates conflicts of inter- est regarding assessment, care, and return to play of concussed athletes (i.e., removing the athletic trainer as beholden to coaches and athletic trainers). This model also considers athletic trainers as allied health care providers in every respect and provides a collaborative and enhanced medical care system for student-athletes. Thompson shares protocols and expectations of the medical model at Princeton University as an exemplar in the diagnosis, assessment, management, and recovery of sport-related concussions. In describing medical model procedures for testing, evaluating, and managing concussions, Thompson provides a compelling argument that cooperative efforts among university health services and athletic department personnel provide an athlete-centered approach that optimizes health and well-being.

In conclusion, the collective papers on concussion management in sport take an interdisciplinary and collaborative approach, featuring perspectives from pediatric, sports medicine, psychology, philosophy, and clinical disciplines. The authors provide an extensive review of the scientific literature and medical consensus statements on concussion management, but also acknowledge that much more research is needed to clarify and inform issues such as diagnosis, assessments, interventions, and protocols for return to school and activity. All the authors featured in this special issue make it apparent that the concept of a concussion management team is essential to address the multiple and complex processes associated with prevention, care, and recovery of concussed athletes. Such teams must include health care providers, kinesiology scholars, school personnel, family members, community stakeholders, and the athletes themselves. I am confident that these scientific, bioethical, and clinical essays will contribute to a greater understanding of the complex nature of sport concussions and increase awareness of how a holistic and collaborative approach to diagnosis, assessment, and care can help reduce prevalence of concussions and promote rapid and effective return to physical activity. 